\title{
Extended Hemi-Hepatectomy with Portal Vein Reconstruction in a Patient with Situs Ambiguous
}

\author{
Keishi Sugimachi ${ }^{a, b} \quad$ Yasuharu Ikeda ${ }^{a} \quad$ Akinobu Taketomi ${ }^{b}$ \\ Morimasa Tomikawa $^{a}$ Katsumi Kawasaki ${ }^{a}$ \\ Daisuke Korenaga $^{a}$ Yoshihiko Maeharab Kenji Takenaka ${ }^{a}$ \\ ${ }^{a}$ Department of Surgery, Fukuoka City Hospital, and bepartment of Surgery and \\ Science, Graduate School of Medical Sciences, Kyushu University, Fukuoka, Japan
}

\section{Key Words}

Situs ambiguous $\cdot$ Hepatocellular carcinoma $\cdot$ Hepatectomy

\begin{abstract}
We report a case of far-advanced hepatocellular carcinoma (HCC) with situs ambiguous, complex visceral and vascular anomalies, who was successfully managed by extended hemi-hepatectomy. A 67-year-old man was referred to our hospital with a large liver mass. Abdominal ultrasonography, computed tomography and angiography revealed $\mathrm{HCC}$ with a diameter of $10 \mathrm{~cm}$, with tumor thrombus in the main and first branch of the portal vein. Multiple complex anomalies in the abdomen were determined preoperatively. He had right-sided spleens-stomach-duodenum, liver at midline, inferior vena cava interruption with azygous continuation, and hepatic arterial anomaly. Extended left lobectomy of the liver with reconstruction of the portal vein was performed. Postoperatively, the patient recovered without major complications, and he was discharged on postoperative day 21 . We report the first successful extended hepatectomy with portal vein reconstruction for HCC in a patient with rare situs anomalies.
\end{abstract}

\section{Introduction}

Hepatectomy has become the standard technique for surgical removal of hepatocellular carcinoma (HCC) [1]. There have been several case reports of the successful application of hepatectomy for HCC patients with situs inversus totalis [2-7], but this is believed to be the first report of major hepatectomy in a patient with situs 
ambiguous, which has more complex visceral and vascular anomalies than situs inversus. We recently encountered a patient with situs ambiguous with polysplenia having a large HCC with tumor thrombus in the main portal trunk, who was successfully managed by hepatectomy with portal vein reconstruction.

\section{Case Report}

A 67-year-old man was referred to our hospital with a large liver mass and was admitted for evaluation. He had been diagnosed and followed with chronic hepatitis $\mathrm{C}$ for 5 years, but had no past history of other serious illness, surgery or hospitalization, and no notable family history. He had never been referred for congenital anomalies. Of the serum tumor markers examined, $\alpha$-fetoprotein and protein induced by vitamin $\mathrm{K}$ absence or antagonist-II were elevated $[42,030 \mathrm{ng} / \mathrm{ml}($ normal $<20 \mathrm{ng} / \mathrm{ml}$ ) and $6,539 \mathrm{mAU} / \mathrm{ml}$ (normal $<40 \mathrm{mAU} / \mathrm{ml}$ ), respectively]. He was found to be serologically positive for anti-hepatitis $C$ virus antibody, and negative for hepatitis B surface antigen.

Computed tomography (CT) showed a $10.5 \times 9.5 \mathrm{~cm}$ tumor in the left lobe by contrast enhancement (fig. 1a). Tumor thrombus was detected in the left first branch and main trunk of the portal vein (fig. 1a). Abdominal CT revealed a right-sided stomach, spleens and truncated pancreas (fig. 1b). The inferior vena cava (IVC) was interrupted, and the left renal vein directly drained to the azygous vein at the thoracic cavity (azygous continuation) (fig. 1c). The right renal vein ascended behind the left lobe of the liver, merged with the hepatic veins and drained to the right atrium of heart. There was no situs anomaly of the heart and lung on chest $\mathrm{X}$ ray. Abdominal angiography showed that the proper hepatic artery diverged from the superior mesenteric artery, and the large tumor in the left lobe, with a hypervascular stain (fig. 2a). Celiac arterial angiography revealed plural splenic arteries and the gastric artery on the right side (fig. $2 \mathrm{~b}$ ). Indirect portography demonstrated a cavernous transformation and collateral vessels. The portal vein could not be stained on portography via the superior mesenteric artery. These findings indicated the presence of a large HCC with a portal thrombus, from the left branch to the main trunk of the portal vein. The visceral anomalies were polysplenia, liver at midline (symmetric liver), right-sided spleens-stomach-duodenum, and truncated pancreas. The vascular anomalies were interrupted IVC, replaced hepatic artery from the superior mesenteric artery, and anomalies of splenic, gastric and pancreatic arteries.

We performed left lobectomy of the liver and portal thrombectomy with reconstruction of the portal vein. Operative findings revealed a large tumor in the left lobe of the liver. Intraoperative ultrasonography showed almost normal anatomy of the intrahepatic bile duct and portal vein. The stomach and duodenum were on the right side of the abdominal cavity (fig. 3 a). Upon entering a hepatoduodenal ligament, common bile duct, hepatic artery proper, and the portal vein with a tumor thrombus, were encircled individually (fig. 3b). The main portal trunk and right portal vein were reconstructed after we removed the tumor thrombus from the portal trunk and left portal vein. The left renal vein was ligated and cut because the vein was located just behind the tumor in the left lobe of the liver. Before ligating the vein, the existence of the collateral vein ascending to the diaphragm was confirmed. There was no sign of renal dysfunction during the postoperative course.

The postoperative course was uneventful. The patient was discharged on postoperative day 21 , and was able to return to work. Pathological examination revealed the size of the tumor as $10.5 \times 9.5 \mathrm{~cm}$, and features of a poorly differentiated HCC with expansive growth. Tumor thrombi with viable carcinoma cells were found in the portal vein.

\section{Discussion}

Situs anomalies are often considered confusing partly because some authors use the terms situs inversus and situs ambiguous interchangeably in the literature. Situs inversus indicates arranged mirror-image location of the viscera. Situs ambiguous is defined as the abnormal arrangement of organs and vessels, as opposed to the orderly arrangement typical of situs inversus [8]. Situs ambiguous is not characterized by a single set of abnormalities but by a spectrum of abnormalities. This case comes under the classification of situs ambiguous with polysplenia (also known as left isomerism or 
bilateral left-sideness). The typical findings of situs ambiguous with polysplenia are as follows: (1) multiple spleens that may be located in either the left or right side of the abdomen, (2) liver and gallbladder at midline, (3) truncated pancreas, (4) IVC interruption with azygous or hemiazygous continuation, (5) right-sided stomach and duodenum, and (6) abnormalities of bowel rotation, although the abnormalities are variable in each case $[8,9]$.

Situs inversus is a rare condition that occurs in one in 5,000-10,000 hospital admissions, and situs ambiguous has a lesser incidence [9]. There has been no report of the definite incidence probably because of its extreme rareness. In this case, the patient was diagnosed and followed for chronic hepatitis type $\mathrm{C}$ and therefore underwent ultrasonography every 6 months for screening for new occurrence of HCC. However, it was difficult to find a tumor at an early stage in this case, probably because of the presence of anatomical anomalies. It is very rare that a patient has situs anomalies, but it must be considered that screening with ultrasonography may overlook a tumor.

Five cases of hepatectomy in situs inversus totalis have been reported [2-6], but there have been no reports of hepatectomy being carried out in patients with situs ambiguous and polysplenia. In all reports, standard hepatectomy was performed for patients with situs inversus totalis. In this case, we performed major hepatectomy with vascular reconstruction for our patient with situs ambiguous, who had a random arrangement of organs.

Full understanding of anatomical mapping prior to surgery and a careful surgical procedure made it possible to successfully and safely perform hepatectomy in our case. The position of the surgeon and surgical devices did not differ from that in normal patients. Mechanical awkwardness was not noted in handling surgical instruments in the reversed spatial situation, and the surgical technique itself did not differ from the usual situation. During operation, recognition of the anatomy was achieved by comparison with preoperative mapping. Operative time and blood loss were comparable to those in orthotopic patients.

In conclusion, careful preoperative anatomical recognition and operative procedure permitted extended hepatectomy with vascular reconstruction in a patient with situs ambiguous. Major surgery for situs ambiguous should be considered a feasible, safe and curative procedure that surgeons should not hesitate to perform. 
Fig. 1. Enhanced abdominal CT. a Large HCC $10 \mathrm{~cm}$ in diameter (arrowheads) and tumor thrombus in a main portal trunk (arrow). b Right-sided stomach and spleens (arrowheads). c Interrupted IVC (white arrow) and left renal vein (black arrow).
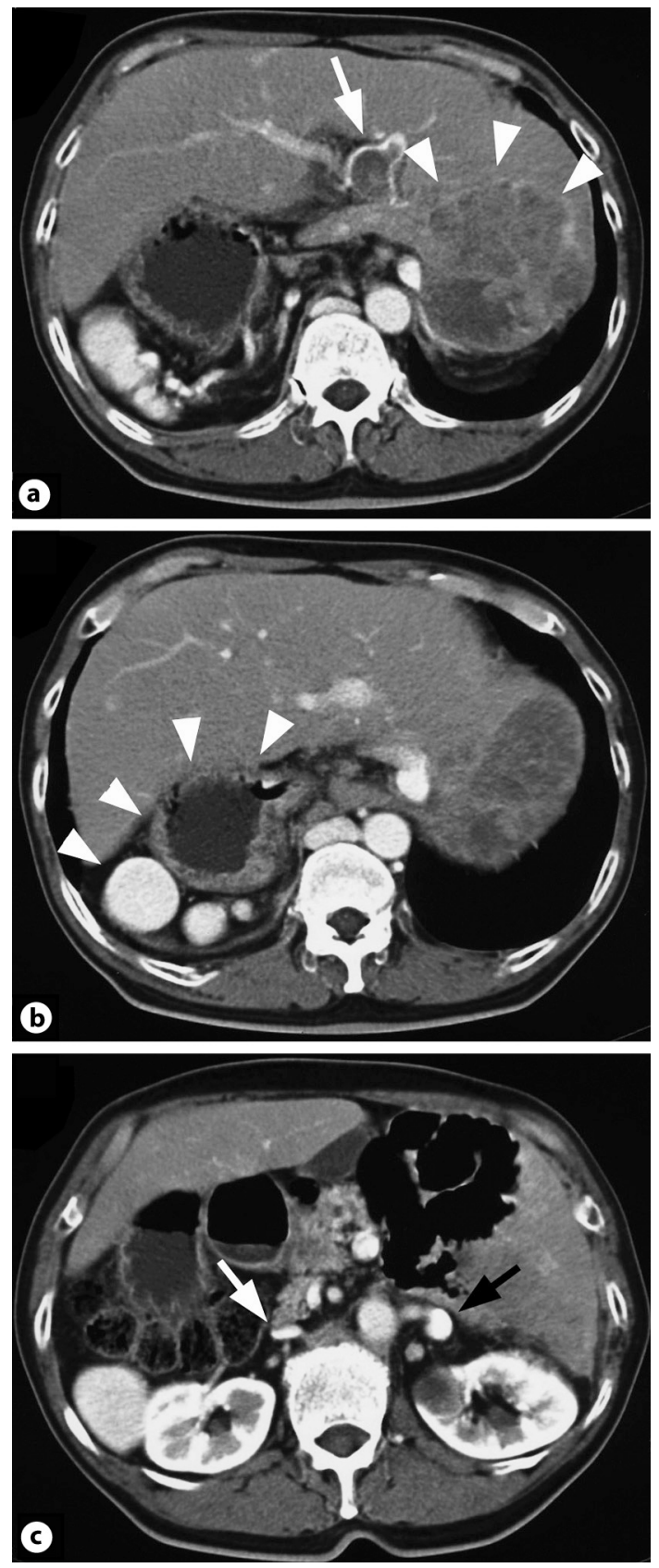
Fig. 2. Abdominal arterial angiography. a Superior mesenteric arterial angiography showed the proper hepatic artery diverging from the superior mesenteric artery (white arrowheads), and the large tumor in the left lobe, with a hypervascular stain (black arrowheads). b Celiac arterial angiography showed plural splenic arteries (white arrowheads) and the gastric artery on the right side (black arrowheads).
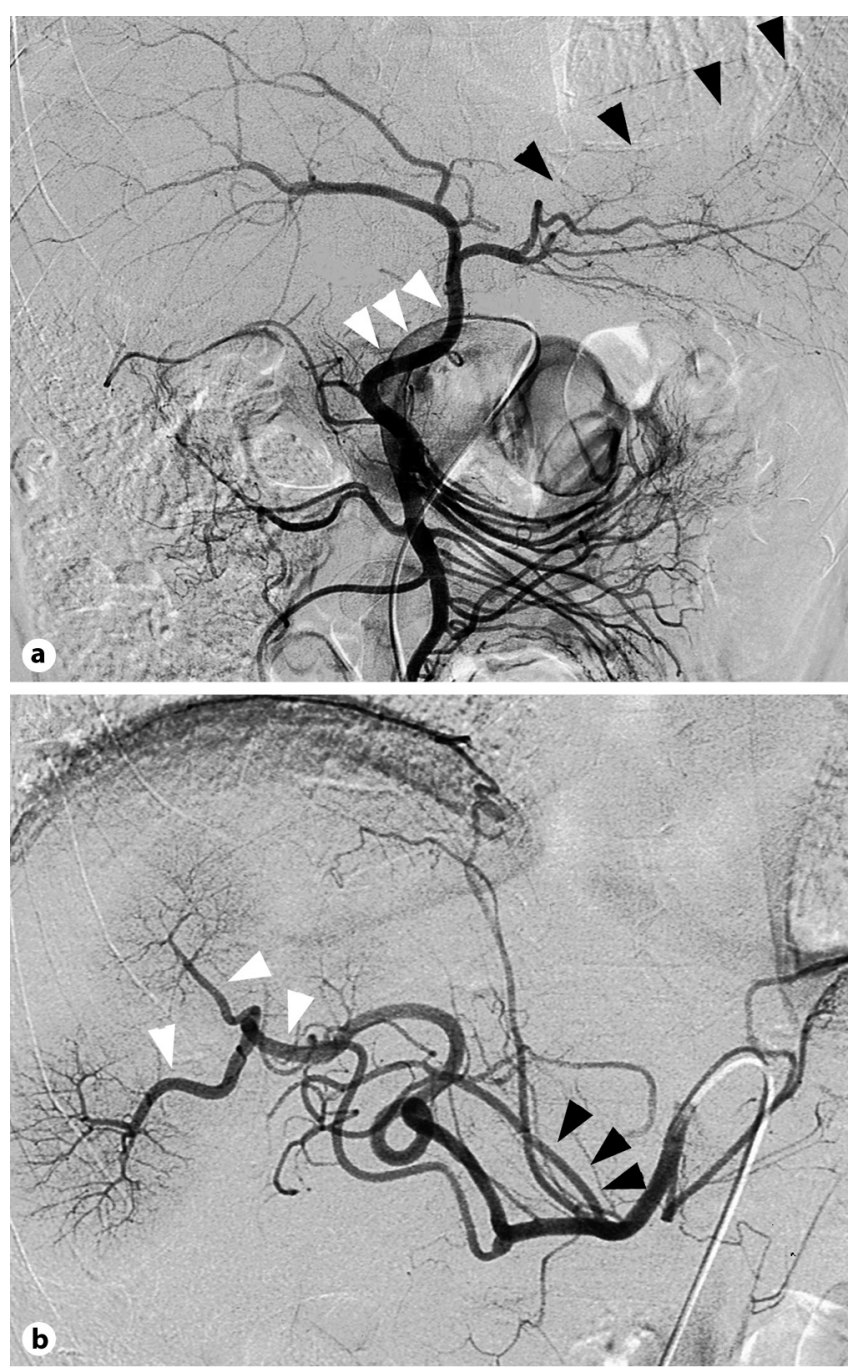
Fig. 3. Intraoperative findings. a The stomach and duodenum were on the right side of the abdominal cavity (arrow). b Upon entering a hepatoduodenal ligament, common bile duct, proper hepatic artery (white arrowheads), and portal vein with tumor thrombus (black arrowheads) were encircled individually.
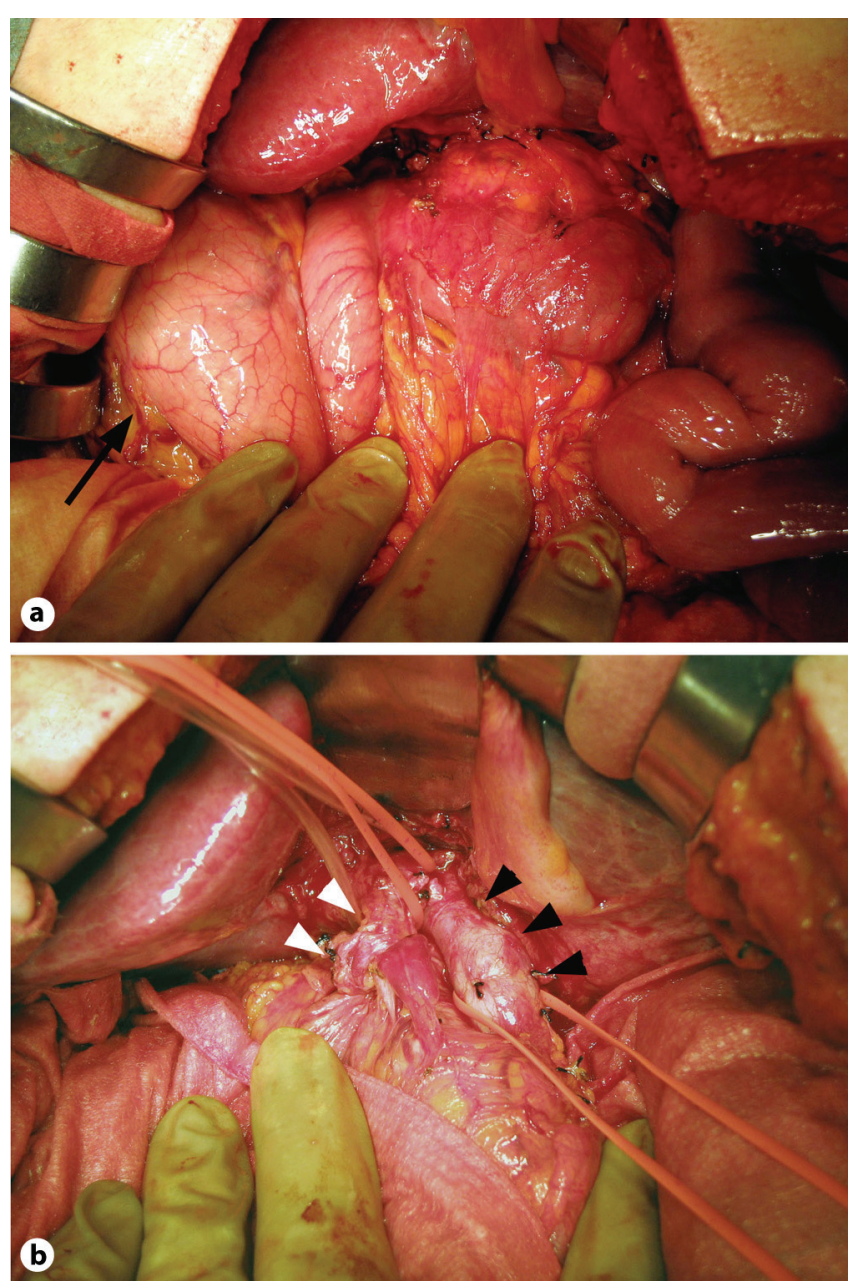


\section{References}

1 Yanaga K: Current status of hepatic resection for hepatocellular carcinoma. J Gastroenterol 2004;39:919-926.

-2 Kanematsu T, Matsumata T, Kohno H, Sugimachi K, Inokuchi K: Hepatocellular carcinoma with situs inversus. Cancer 1983;51:549-552.

-3 Sano T, Kamiya J, Nagino M, Kanai M, Uesaka K, Nimura Y: Hepatectomy for proximal bile duct carcinoma in a patient with situs inversus; a case report. Hepatogastroenterology 2003;50:1266-1268.

-4 Niki Y, Shiraki K, Enokimura N, Okano H, Yamanaka T, Takase K, Nakano T: Hepatocellular carcinoma associated with situs inversus totalis. J Clin Gastroenterol 2004;38:382-383.

5 Kakinuma D, Tajiri T, Yoshida H, Mamada Y, Taniai N, Kawano Y, Mizuguchi Y, Shimizu T, Takahashi T, Akimaru K, Aramaki T, Takano T: A case of hepatocellular carcinoma with situs inversus totalis. J Nippon Med Sch 2004;71:209-212.

-6 Kamiike W, Itakura T, Tanaka H, Hatanaka N, Nakamuro M, Miyata M, Izumi H: Hepatic segmentectomy on primary liver cancer with situs inversus totalis. HPB Surg 1996;9:169-172; discussion 172-173.

7 Kim YI, Tada I, Kuwabara A, Kobayashi M: Double cancer of the liver and stomach with situs inversus totalis - a case report. Jpn J Surg 1989;19:756-759.

-8 Fulcher AS, Turner MA: Abdominal manifestations of situs anomalies in adults. Radiographics 2002;22:1439-1456.

-9 Applegate KE, Goske MJ, Pierce G, Murphy D: Situs revisited: imaging of the heterotaxy syndrome. Radiographics 1999;19:837-852; discussion 853-854. 